\title{
Image segmentation based on scaled fuzzy membership functions
}

\author{
Jantzen, Jan; Ring,, P.; Christiansen, Pernille
}

Published in:

Proceedings of the 2nd IEEE International Conference on Fuzzy Systems

Link to article, DOI:

10.1109/FUZZY.1993.327401

Publication date:

1993

Document Version

Publisher's PDF, also known as Version of record

Link back to DTU Orbit

Citation (APA):

Jantzen, J., Ring, P., \& Christiansen, P. (1993). Image segmentation based on scaled fuzzy membership functions. In Proceedings of the 2nd IEEE International Conference on Fuzzy Systems (Vol. Volume 2, pp. 714718). IEEE. https://doi.org/10.1109/FUZZY.1993.327401

\section{General rights}

Copyright and moral rights for the publications made accessible in the public portal are retained by the authors and/or other copyright owners and it is a condition of accessing publications that users recognise and abide by the legal requirements associated with these rights.

- Users may download and print one copy of any publication from the public portal for the purpose of private study or research.

- You may not further distribute the material or use it for any profit-making activity or commercial gain

- You may freely distribute the URL identifying the publication in the public portal 


\title{
Image Segmentation Based on Scaled Fuzzy Membership Functions
}

\author{
Jan Jantzen, MIEEE \\ Technical University of Denmark \\ Building 325, DK-2800 Lyngby, DENMARK \\ Bitnet/eam: stardust@vm.uni-c.dk \\ Poul Ring, MIEEE, and Pernille Christiansen \\ Danish Research Centre of Magnetic Resonance \\ Hvidovre Hospital, Kettegaard Alle 30 \\ DK-2650 Hvidovre, DENMARK
}

\begin{abstract}
As a basis for an automated interpretation of magnetic resonance images, the paper proposes a fuzzy segmentation method. The method uses five standard fuzzy membership functions: small, small medium, medium, large medium, and large. The method fits these to the modes of interest in the image histogram by means of a piece-wise linear transformation. A test example is given concerning a human head image, including a sensitivity analysis based on the fuzzy area measure. The method provides a rule based interface to the physician.
\end{abstract}

\section{INTRODUCTION}

Physicians at the Hvidovre hospital measure the volume of objects inside patients' heads using magneto-resonance (MR) images. The physician gets an MR image on the computer screen and draws out regions of interest using mouse and cursor. The computer calculates the area which is then used to calculate the volume of an object. The image in Fig. 1 is an example of a slice of the human head. The slice is 4 millimeters thick and passes through the nose, the eyes, and the ears.

This task becomes formidable, if the objective is, say, to measure the volume of the brain in a hundred patients with fifteen slices per patient. An automatic system that finds the regions of interest and measures the area would be a considerable help. The aim of this paper is to provide a robust segmentation method with a rule based interface as a basis for such a system.

An input image is giveri as a matrix of pixel amplitudes. The general rule of thumb is that small amplitudes indicate fluids, medium amplitudes indicate brain tissue, and large amplitudes indicate dense tissue, such as fat and bone marrow. Each pixel class occupies a contiguous range in the histogram of pixel amplitudes. The histogram contains several peaks (modes), and the distributions overlap indicating that class boundaries are fuzzy. The separation of amplitudes is clearly an amplitude segmentation problem, and it falls into the category of thresholding and pixel classification problems (Bezdek \& Pal, 1992).

There exists a method for obtaining fuzzy and non-fuzzy regions in a bimodal image (Pal \& Rosenfeld, 1988). The method ingeniously optimizes compactness and fuzziness using a single s-shaped membership function to find the minimum between the two peaks in the histogram. Compactness is one of several useful geometrical measures (Rosenfeld, 1984; Pal \& Ghosh, 1992), that can help in the segmentation. Sume generalisation of the method is needed, though, to apply it to the multi-modal problem at hand.

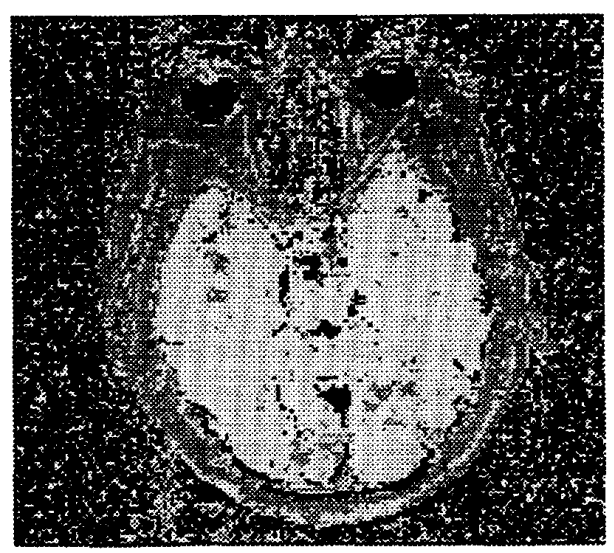

Fig. 1 Three-level magnetic resonance image of human head; roughly speaking, light grey = brain tissue, grey $=$ fatty tissue, dark grey = fluids. The background is a mixture. 
There also exists a rule-based system for analysis of MR images in three dimensions (Raya, 1990). It computes a confidence function and confidence levels indicating membership of voxel classes. A voxel at the center of a class, that is, where the histogram has a peak, is assigned the maximum confidence of 1 ; monotonically decreasing levels are assigned to the voxels on both sides of the peak based on the shape of the population distribution. The system uses concepts that are similar to fuzzy sets, but it does not apply fuzzy sets explicitly.

The problem at hand requires a rough segmentation with latitude for small variations, say, $\pm 10 \%$ in pixel amplitudes as well as object positions and sizes. The method presented in the following uses fuzzy membership functions to cope with variations.

\section{SEGMENTATION METHOD}

The idea is to fit a fuzzy membership function to each amplitude interval of interest in the histogram. Thus fuzzy labels, such as small, medium and large, can be associated with each interval of interest. The goal is to perform the segmentation using rules based on the fuzzy labels.

There are, as usual, two design questions to consider: How does one determine the shape of the fuzzy sets, and how many sets are necessary and sufficient? The first question has been dealt with in a particular case, namely, the fuzzy controller for cement kilns (Holmblad \& Østergaard, 1982). The trick is to scale the incoming measurements according to some piece-wise linear function, before the membership values are looked up in standard membership functions defined on a standard universe. As a result the controller's collection of primary membership functions has not been altered during its ten years of existence.

With regard to the second design question, we have defined a family of five standard, or primary, sets on a stan-

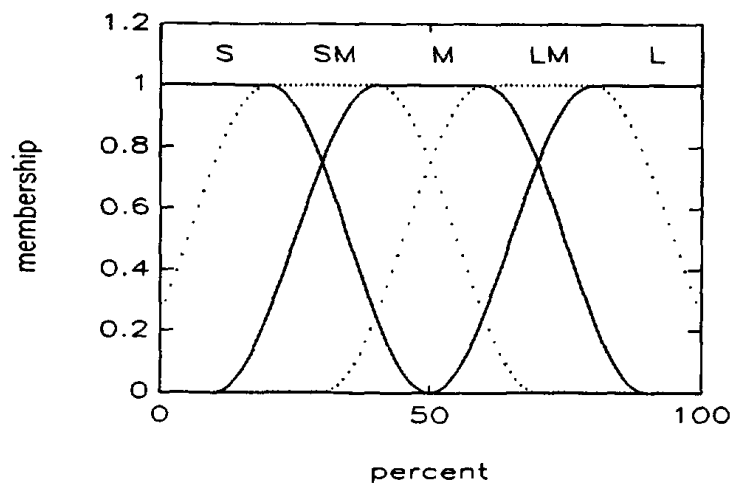

Fig. 2 Definition of fuzzy sets. Solid: small (S), medium (M), large (L). Dotted: small medium (SM) and large medium (LM). dard universe: small, small medium, medium, large medium and large (Fig. 2). They are continuous functions based on the cosine function. These sets cover the universe in such a way, that any element is a full member of at least one primary set. The universe is the closed interval $[0,100]$ corresponding to percentages of full range. The width of the flat peaks has been designed to accomodate $\pm 10 \%$ latitude. The reason for choosing cosine, instead of the more usual exponential or power functions, is convenience; it can be applied as a building-block in all primary sets (see the appendix for definitions).

More sets can be derived in the usual manner from any primary sets $\mathbf{A}$ and $\mathbf{B}$ when needed, e.g., not $\mathbf{A}(1-\mathbf{A}), \mathbf{A}$ and $B(\min (A, B)), A$ or $B(\max (A, B))$, very $A\left(A^{2}\right)$, and more or less $A\left(A^{1 / 2}\right)$.

Since the fuzzy sets are defined on a standard universe, usually different from the universe of image amplitudes, a scaling is needed to associate the amplitudes in the image with the universe of the fuzzy sets (Fig. 3). We have therefore defined a piece-wise linear function with breakpoints corresponding to anchor-points in the fuzzy primary sets. For a given image, the scaling function $\sigma$ relates amplitudes $\mathbf{A}$ in the input image to percentages $\mathbf{P}$ on the standard universe, by

$$
P=\sigma(A)
$$

An expert has to decide on the intervals of interest in the histogram, but, if it has a mode within such an interval with a valley on each side, the peak and the valleys should be associated with a breakpoint each. The scaling function ensures that the valleys in the histogram are mapped onto the crossover points between neighbouring membership functions, and that the peak is mapped onto the peak of a membership function. For example, in Fig. 4, the second mode is mapped onto the set medium by associating the left valley at $a_{2}$ with the cross-over point between medium and small (at $30 \%$ on the standard universe cf. Fig. 2), the peak at $a_{3}$ with the center of medium (at $50 \%$ ), and the right valley at $a_{4}$ with the cross-over point between medium and large (at $70 \%$ ).

Recall that a fuzzy membership function $\mu$ relates percentages to membership values $\mathbf{M}$, that is,

$$
M=\mu(P)
$$

where $\mu$ is the membership function for small, medium, or large, for instance. The composition of (1) and (2) is

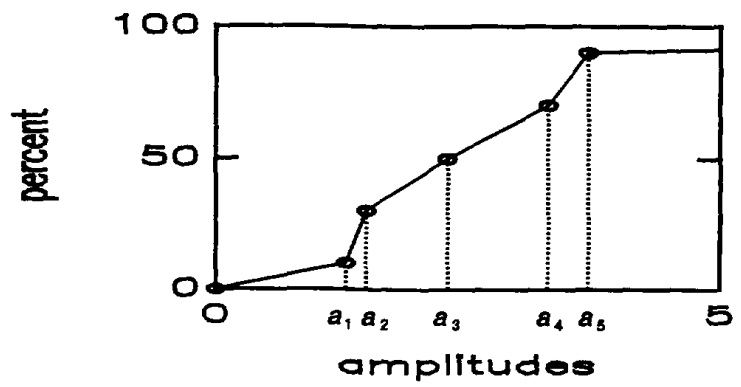

Fig. 3 Scaling function for the head image. 


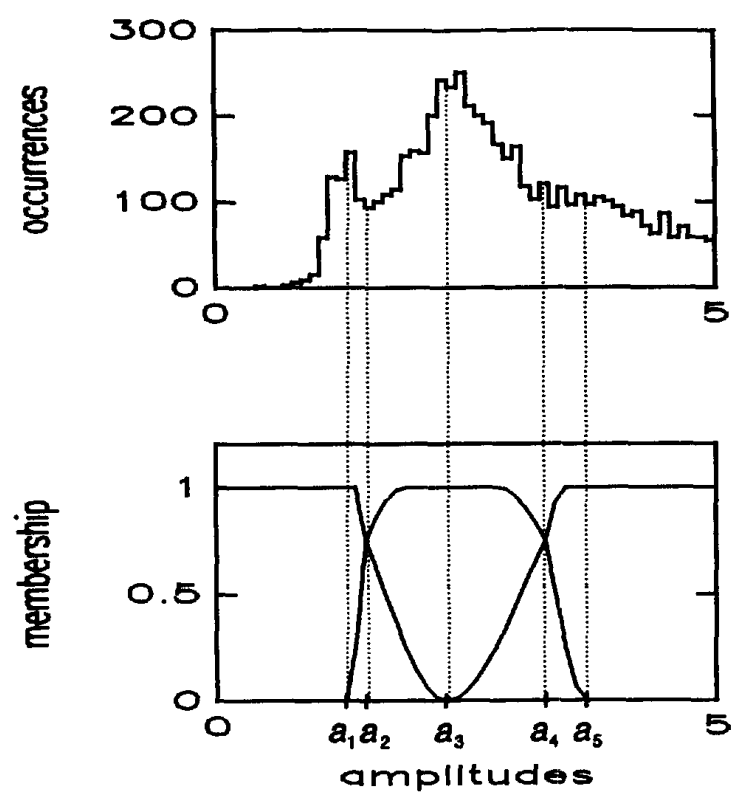

Fig. 4 Histogram of eye region with background suppressed (top), and the result of scaling the sets small, medium, large (bottom).

$$
M=\mu(\sigma(A))
$$

Our goal is to find the matrix of membership values $\mathbf{M}$. Rather than forming a symbolic expression for the composed function $\mu \circ \sigma$, which is equivalent to the scaled membership function, it is easier in practice to compute $\sigma(A)$ for the whole image and insert the result into $\mu$. The computation of $\sigma(A)$ can be done in a pre-processing step, in order to bring down the response times during run-time.

Conceptually, segmentation rules are in the familiar IFTHEN format, for example,

\section{IF $P$ is small THEN region is background.}

The actual implementation deviates from this format, partly because of the syntax of the computer language used, partly because the rules are so simple. The expression for the above rule is rather

$$
\text { background }=\operatorname{small}(P)
$$

The example in the next section, will show how two rules look in the Matlab language (Mathworks, 1990).

Summing up, the total segmentation procedure can be described in 7 steps:

1. define primary sets;

2. compute histogram;

3. identify anchor-points;
4. create scaling function $\sigma$;

5. scale image using (1);

6. make rules;

7. execute rules using scaled image.

It is important to realize that the method is intended for screening of many, more or less similar images, that is, the same slice from many patients, and step 3 is performed once for the whole sequence by a domain expert.

The last step is intended to be followed by an object recognition step, possibly based on non-fuzzy features (centroid, area, perimeter, compactness, clustering) or fuzzy geometric features (area, compactness, height, width, adjacency, axes, density; Pal \& Ghosh, 1992) - but that is outside the scope of this paper. The following example demonstrates some details of the procedure.

\section{TEST EXAMPLE AND DISCUSSION}

The test image (Fig. 1) is a calculated image. For each slice the MR scanner produces several images representing different features, for example proton density and the relaxation times $T_{1}$ and $T_{2}$ (e.g. Hinshaw \& Lent, 1983). The test image is the ratio of two socalled spin-echo images (repetition time $T_{r}=1.8 \mathrm{secs}$, and echo times $T_{c}=30$ and 90 secs). The scanner is a Siemens SP63/84, and the magnetic field is 1.5 Tesla. In a prototype of a segmentation system, the data files are imported via ascii files into Matlab v. $3.5 \mathrm{~g}$ on a $\mathrm{PC} / 386$. The image comes in a $256-$ by- 256 matrix, but is reduced to 181 -by-181 by clipping some of the background.

The pixel amplitudes are positive integers corresponding to the damping of the echo over time. Larger numbers indicate larger damping, and this in turn indicates denser matter; that is why small amplitudes indicate fluids, medium amplitudes indicate brain tissue, and large amplitudes indicate dense tissue.

Accordingly, the histogram has three intervals of interest. The two lowest intervals are identified in Fig. 4 as two distinct modes. The third interval of interest is difficult to see because of overlap with the neighbouring interval. In the order of increasing amplitudes, we associate the labels small, medium, and large with the three interesting intervals.

The scaling function $\sigma$ in Fig. 3 scales the three corresponding fuzzy sets, so that they fit the intervals of interest (Fig. 4). After scaling the whole image, as in (1), the result is a matrix of real numbers between zero and one hundred corresponding to the standard universe. Any pixel's membership of any fuzzy set can now be looked up in the membership functions.

Suppose we are searching for the left eye, only a few rules are sufficient. The eyes are filled with fluid, so the pixel amplitudes are small or, perhaps, small medium due to overlap into the next interval of interest. The position in the image is upper slightly left as defined in Fig. 5 corresponding 


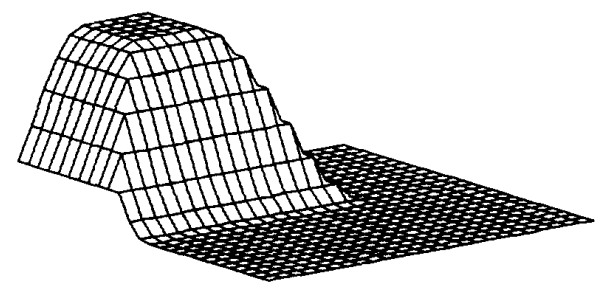

Fig. 5 The 2-dimensional fuzzy set "upper slightly left".

to upsleft in the appendix. Assuming the background is determined by other means, the following two rules in the Matlab language define the region around the eye

fluid $=$ and $($ or $($ swall $(P)$, snediur $(P)), \operatorname{not}($ background $)) ;$

lefteye $\square$ and(fluid,upsleft);

The result lefteye is a 181-by-181 matrix of fuzzy membership values. Its highest valued pixels have the highest compatibility with the rules. Fig. 6 shows lefteye at different $\alpha$ cuts, that is, each picture shows the pixels with memberships $\mathbf{M} \geq \alpha$.

The figure shows that the set upsleft reduces the region of interest to a fuzzy window. It appears that the eye is determined completely at $\alpha=1$. This is, however, partly a coincidence. Generally, the resultant segment may contain the object, as well as some other objects. A fuzzy adjacency measure (Pal \& Ghosh, 1992) and connectedness can be applied at this stage, in order to distinguish the eye from other objects by area and perimeter.

The critical step in the procedure is the scaling. If the breakpoints in the scaling function are misplaced, the region

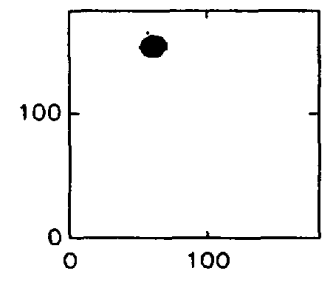

(o) membership $=1$

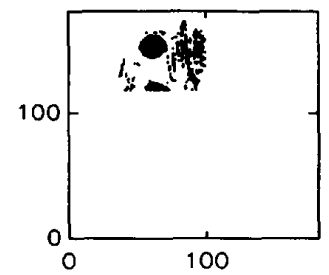

(c) membership $>=0.5$

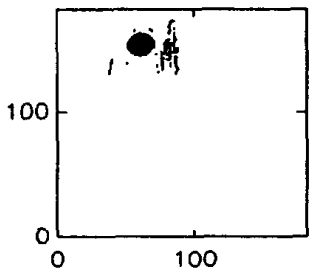

(b) membership $>=0.8$

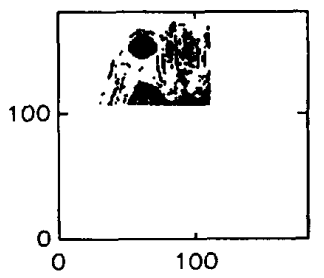

(d) membership $>=0.2$
Fig. 6 The fuzzy set "lefteye" at different membership levels (alpha-cuts). of interest may get membership values less than 1 in the final fuzzy set. Given the primary sets, the sensitivity depends on the widths and the positions of the scaled membership functions. Thus, the sensitivity is related to the cross-over points and peak points in the scaled membership functions. To get a quantitative indication of the robustness, we have used the fuzzy area, $a(\mu)=\Sigma_{\mu}$ (Rosenfeld, 1984) in two experiments on the head image data.

In the first experiment, the cross-over point $a_{2}$ between the sets small and medium was moved from $a_{1}$ to $a_{3}$ over the valley between the neighbouring peak points. As $a_{2}$ moves, the area a(small and medium) varies (Fig. 7, left). The area can be perceived as a measure of ambiguity, in the sense that it measures the area of the transition zone between the sets small and medium. Ideally, this area should be minimal, which is the case when $a_{2}$ corresponds to the valley in the histogram. From the figure, it can be measured that a change of $\pm 10 \%$ from the optimum, implies at most $7 \%$ change of area relative to the maximal range of change. The change is less than $5 \%$ relative to the absolute area.

In the second experiment, the peak amplitude $a_{3}$ of the scaled set medium was moved from $a_{2}$ to $a_{4}$ over the peak in the histogram (Fig. 7 , right). The area a(medium) can be perceived as a measure of the goodness of the fit. Ideally, this area should be maximal, which is the case when $a_{3}$ corresponds to the peak in the histogram. From the figure, it can be measured that a change of $\pm 10 \%$ from the optimum, implies at most $9 \%$ change of area relative to the maximal range of change. The change is less than $1 \%$ relative to the absolute area.

This is so far regarded as satisfactory, since the response is within the (arbitrary) design margin of $\pm 10 \%$. Both curves are remarkably smooth with a distinct optimum. This could be utilized to automatically adjust the break-points of the scaling function instead of a manual tuning (cf. Pal \& Rosenfeld, 1988).

The example went well in the case of the eye, because it is a uniform and homogeneous object. It is difficult, however, to find the nose and the ears, because the method is not suited for line type objects. In the case of the brain, it is possible to find a segment containing the whole brain, but it contains holes due to objects inside, such as patches of
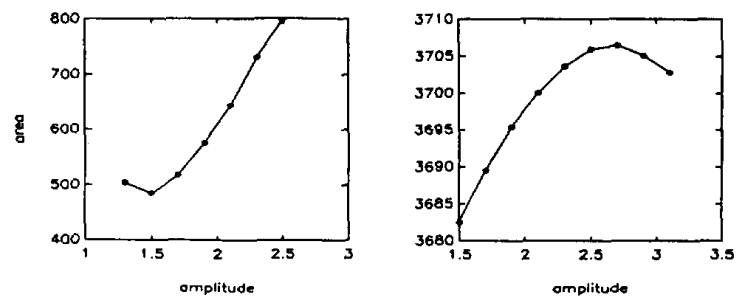

Fig. 7 Results from sensitivity analysis. Area of overlap between small and medium (left), and area of medium sized pixels (right). 
fluid. Smaller objects, like the cerebellum and the ventricles are better targets for the method.

\section{CONCLUSION}

The proposed segmentation method is designed for greyscale, multi-modal images with overlapping individual distributions. The method is developed for screening of many images that are more or less similar. In comparison with the method by Pal \& Rosenfeld (1988), the proposed method generalises to multi-modal images, and in comparison with the rule-based system by Raya (1990), the proposed method is on the more firm foundation of fuzzy sets. Furthermore, the method uses essentially the scaling mechanism of Holmblad \& Østergaard (1982) in order to standardize the definition of fuzzy membership functions. The method may be regarded as a segmentation method which may precede region growing and edge detection steps in an object recognition system. It is characteristic that this method provides a rule based interface.

The further plans are to extend to three dimensions and then analyse pathologies. A perspective is to have the physician design and input rules for object extraction, independent of the domain expert.

\section{ACKNOWLEDGMENT}

Much clever groundwork has been done by Søren Vejgaard-Nielsen, Pall Vesturbu, Poul Mols and Steen Astman Jensen in their M.Sc. projects.

\section{REFERENCES}

[1] Bezdek, J.C. \& S.K. Pal, eds.: "Fuzzy Models for Pattern Recognition." New York: IEEE Press, 1992 (selected reprints volume).

[2] Hinshaw, W.S. \& A.H. Lent: "An Introduction to NMR Imaging: From the Bloch Equation to the Imaging $\mathrm{E}$ quation." Proc. IEEE, 1983, 71(3), 338 - 350.

[3] Holmblad, L.P. \& J.-J. Østergaard: "Control of a cement kiln by fuzzy logic". In: M.M. Gupta \& E. Sanchez (Eds): "Fuzzy Information and Decision Processes." Amsterdam: North Holland, 1982, 389-399.

[4] Mathworks, The: "Matlab - User's Guide." The MathWorks, Inc., Cochituate Place, 24 Prime Park Way, Natick, MA 01760, USA, 1990.

[5] Pal, S.K. \& A. Ghosh: "Fuzzy Geometry in Image Analysis." Fuzzy Sets and Systems, 1992, 48(1), 23 - 40.
161 Pal, S.K. \& A. Rosenfeld: "Image Enhancement and Thresholding by Optimization of Fuzzy Compactness." Pattern Recognition Letters, 1988, 7, 77-86. Rpt. in Bezdek \& Pal, 1992, pp. 369 - 378.

[7] Raya, S.P.: "Low-Level Segmentation of 3-D Magnetic Resonance Brain Images - A Rule Based System." IEEE Trans, on Medical lmaging, 1990, 9(3), 327 - 337.

[8] Rosenfeld, A.: "The Fuzzy Geometry of Image Subsets." Pattern Recognition Letters, 1984, 2, 311 - 317. Rpt. in Bezdek \& Pal, 1992, pp. 340 - 346.

\section{APPENDIX: Definitions}

The primary fuzzy sets are defined using a cosine function. The s-curve is defined by the equation

$s\left(x_{0}, b, x\right) \otimes \begin{cases}0 & , x<x_{0}-b \\ 1 / 2+1 / 2 \cos \left(\frac{x-x_{0}}{b} \pi\right) & , x_{0}-b \leq x \leq x_{0} \\ 1 & , x>x_{0}\end{cases}$

where $x_{0}$ is the coordinate of the peak, $x$ is the independent variable (image amplitudes), and $b$ is the width of the "proportional" band, i.e., the section with a slope. The inverted s-curve is defined

$z\left(x_{0}, b, x\right) \triangleleft \begin{cases}1 & , x<x_{0} \\ 1 / 2+1 / 2 \cos \left(\frac{x-x_{0}}{b} \pi\right) & , x_{0} \leq x \leq x_{0}+b \\ 0 & , x>x_{0}+b\end{cases}$

From these, all other membership functions are derived:

$\pi\left(x_{1}, x_{2}, b, x\right) \Delta \min \left(s\left(x_{1}, b, x\right), z\left(x_{2}, b, x\right)\right)$

$\operatorname{medium}(x) \backsim \pi(40,60,30, x)$

small $(x) \wedge \pi(0,20,30, x)$

large $(x) \wedge \pi(80,100,30, x)$

$\operatorname{smedium}(x) \wedge \pi(20,40,30, x)$

$\operatorname{lmedium}(x) \wedge \pi(60,80,30, x)$

and $(x, y) \Delta \min (x, y)$

upper $(x) \triangle \operatorname{small}(x)$

sleft $(x) \triangle \operatorname{smedium}(x)$

upsleft $(x) \triangle \underset{i, j}{\operatorname{AND}}\left(\right.$ upper $\left(x_{i}\right)$, sleft $\left.\left(y_{j}\right)\right)$ 